\title{
Caractérisation Des Boues De Sucrerie Et Des Argiles Pour La Neutralisation Des Résidus Miniers Acides De La Mine De Kettara (Jebilet Centrales, Maroc)
}

\author{
Youssef Zerhouni \\ Saida Alikouss \\ Najib Saber \\ Samiha Nfissi \\ Ghalem Zahour \\ Zouhir Baroudi \\ Mohamed Samir
}

Laboratoire de Géodynamique des Chaines Anciennes (LGCA)

Département de Géologie, Faculté des Sciences Ben M’Sik,

Université Hassan II de Casablanca

doi: 10.19044/esj.2016.v12n15p321 URL:http://dx.doi.org/10.19044/esj.2016.v12n15p321

\begin{abstract}
The minerals in the tailings, subject to the action of water and atmospheric oxygen, can generate Acid Mine Drainage (AMD). The latter is considered the most important environmental issue facing the mining industry. Its environmental impacts include the destruction of the flora and fauna in infected rivers and contamination of groundwater.

The abandoned mine site Kettara, located about $32 \mathrm{~km}$ northwest of Marrakech (Morocco), chosen as a pilot site for this study, more than 3 million tons of tailings stored at ground level without any concern for their environmental consequences.

These solid residues, with high concentrations of heavy metals (As, Pb, Fe, $\mathrm{Cu} \ldots)$, produce leachate very acidic $(\mathrm{pH}<2.9)$ may contaminate the water resources of the region.

To mitigate the DMA phenomenon in this mining site, different protocols have been proposed using candy sludge (Mud Pulp Sweets: MPS), rich in carbonates from the Moroccan Sugar Company Unit (COSUMAR) and red clays (Clays: CLY) of the city of Safi.

Physicochemical characterization and mineralogical of these two materials was performed an provided promising results regarding the effectiveness of the use of alkaline materials in the stabilization of tailings Kettara.
\end{abstract}


Indeed, these starting materials are provided with a net neutralizing power which is of the order of $878.5 \mathrm{~kg} \mathrm{CaCO3} \mathrm{/} \mathrm{t} \mathrm{for} \mathrm{MPS} \mathrm{and} 299 \mathrm{~kg} \mathrm{CaCO3} \mathrm{/} \mathrm{t}$ for CLY.

The valuation of MPS and CLY, as a main component of an alkaline amendment, is likely to significantly reduce the effects of DMA in this semiarid climate mine.

Keywords: acid mine drainage, candy sludge, clay, Kettara, alkaline amendment.

\section{Résumé}

Les minéraux présents dans les rejets miniers, soumis à l'action de l'eau et de l'oxygène atmosphérique, peuvent générer du Drainage Minier Acide (DMA). Ce dernier est considéré comme le plus important problème environnemental auquel fait face l'industrie minière. Ses impacts sur l'environnement incluent la destruction de la flore et de la faune aquatique dans les cours d'eau infectés ainsi que la contamination de l'eau souterraine.

Le site minier abandonné de Kettara, situé à environ 32 km au Nord-Ouest de Marrakech (Maroc), choisi comme site pilote pour cette étude, compte plus de 3 millions de tonnes de résidus miniers entreposés à même le sol sans aucun souci de leurs conséquences environnementales.

Ces résidus solides, à fortes concentrations en métaux lourds (As, Pb, Fe, $\mathrm{Cu} . .$.$) , produisent des lixiviats très acides (\mathrm{pH}<2.9)$ susceptibles de contaminer les ressources hydriques de la région.

Afin d’atténuer le phénomène de DMA dans ce site minier, différents protocoles ont été proposés en utilisant les boues de sucrerie (Mud Pulp Sweets : MPS), riches en carbonates provenant de la Compagnie Sucrière Marocaine, Unité de Casablanca (COSUMAR) et les argiles (Clays : CLY) rouges de la ville de Safi.

Une caractérisation physico-chimique et minéralogique de ces deux matériaux a été effectuée et a fourni des résultats prometteurs quant à l'efficacité de l'utilisation de ces matériaux alcalins dans la stabilisation des rejets miniers de Kettara.

En effet, ces matériaux initiaux sont dotés d'un pouvoir net de neutralisation qui est de l'ordre de $878.5 \mathrm{~kg} \mathrm{CaCO}_{3} / \mathrm{t}$ pour les MPS et de $299 \mathrm{~kg} \mathrm{CaCO}_{3} / \mathrm{t}$ pour les CLY.

La valorisation des MPS et des CLY, en tant que composante principale d'un amendement alcalin, est susceptible de diminuer considérablement les effets du DMA dans cette mine à climat semi-aride.

Mots clés : Drainage minier acide, boues de sucrerie, argiles, Kettara, amendement alcalin. 


\section{1- Introduction}

Au Maroc, l’activité minière a été au centre des enjeux industriels depuis plusieurs siècles. Elle apporte des perspectives économiques aux populations locales. Cependant, elle entraîne une modification profonde de l'environnement. Les enjeux environnementaux associés à l'exploitation minière sont nombreux. Le minerai extrait d'une mine subit plusieurs étapes de concassage, de concentration et de traitement. De ce fait, toutes les étapes de traitement, d'extraction et séparation métallurgiques, produisent des rejets miniers solides et liquides pouvant contenir des quantités parfois non négligeables en minéraux métalliques non valorisables. Ces derniers, exposés à l'air et à l'eau, causent un grand changement dans les conditions de stabilité des substances chimiques. Généralement, ce changement produit une oxydation des sulfures générateurs d’acidité. Autrement, le $\mathrm{pH}$ diminue ce qui favorise, par ailleurs, la solubilité de certains métaux. Ce phénomène, bien connu, est nommé Drainage Minier Acide (DMA). Il peut être amplifié par la présence de bactéries qui prospèrent quand les $\mathrm{pH}$ deviennent très bas ( pH 3). Les eaux contaminées par le DMA peuvent provenir de divers types d'exploitation, incluant des mines de métaux précieux (Au, Ag...), de métaux de base (Cu, Ni, Zn, Pb...), de charbon et d'uranium (Plante, 2004). Depuis quelques années, la majorité des pays industrialisés imposent des règles environnementales de plus en plus strictes intervenant pendant et après exploitation d'un site minier, ainsi que la préparation d'un plan de restauration final dès l'étude de faisabilité d'un projet (e.g. Directive 019 MDDEP 2012). De ce fait, plusieurs méthodes d'atténuation peuvent être utilisées pour prévenir la production de DMA dans les parcs à résidus miniers, parmi les quelles le dépôt subaquatique (Aubertin et al., 2002), les recouvrements de type couvertures à effets de barrière capillaire (Dagenais et al., 2003; Bossé, 2013), la désulfuration environnementale (Benzaazoua et Kongolo, 2003 ; Mermillod-Blondin et al., 2005) et l'amendement alcalin qui consiste à mélanger les résidus générateurs de DMA avec des matériaux alcalins afin de limiter l'oxydation des sulfures et de neutraliser l'acidité générée (Hakkou et al,. 2009; González et al., 2012; Nfissi et al., 2014).

Cette étude vise l'utilisation des sous-produits alcalins fins (Les boues de sucrerie (MPS) et les argiles (CLY) en vue de la réduction de l'impact du DMA dans le site de Kettara (Maroc). L'étude des propriétés physiques, chimiques et minéralogiques des boues de sucrerie et des argiles sera entamée pour déterminer leurs pouvoirs neutralisant afin d'approuver les scénarios les plus appropriés pour le contrôle de ce phénomène dans ce site.

La mine de Kettara est située à environ $32 \mathrm{~km}$ au Nord-Ouest de la ville de Marrakech, dans le massif de Jebilets (Fi. 1). 
Elle correspond à un amas sulfuré (Viséen supérieur), caractérisé par une minéralisation constituée essentiellement de pyrrhotine et de pyrite, mais appauvrie en métaux de base (Bordonaro, 1984). La structure minéralisée de Kettara, de forme lenticulaire, est subverticale et sécante à la stratification, s'étend sur $1500 \mathrm{~m}$, suivant une direction N30. Elle est de puissance variable de 0 à $70 \mathrm{~m}$, et s'enracine sur plus de $500 \mathrm{~m}$.

Les principales caractéristiques gîtologiques de l'amas sulfuré de Kettara sont rattachées à sa position stratigraphique au dessus du volcanisme acide du membre supérieur d'une série dite de Sarhlef (Jbilets centrales) et en dessous d'une autre dite de Teksim (Jbilets orientales).

La minéralisation présente des structures primaires témoignant de sa contemporanéité avec la sédimentation. Le contrôle structural joue un rôle important dans les positions du corps minéralisé avec développement d'une altération hydrothermale importante à séricite, chlorite, qui accompagne la mise en place de l'amas (Felenec et al., 1986).

La mine de Kettara a été exploitée pendant 35 ans, au début pour les ocres de 1945 à 1961, ensuite pour la pyrite et le cuivre de 1956 à 1963 essentiellement dans la zone de cémentation et enfin pour la pyrrhotine de 1965 à 1980.

Depuis plus d'une trentaine d'années, l'abandon total sans réhabilitation de cette mine a entraîné le dépôt de 3 millions de tonnes de rejets miniers sur une superficie de 16 hectares (Hakkou et al., 2008a).

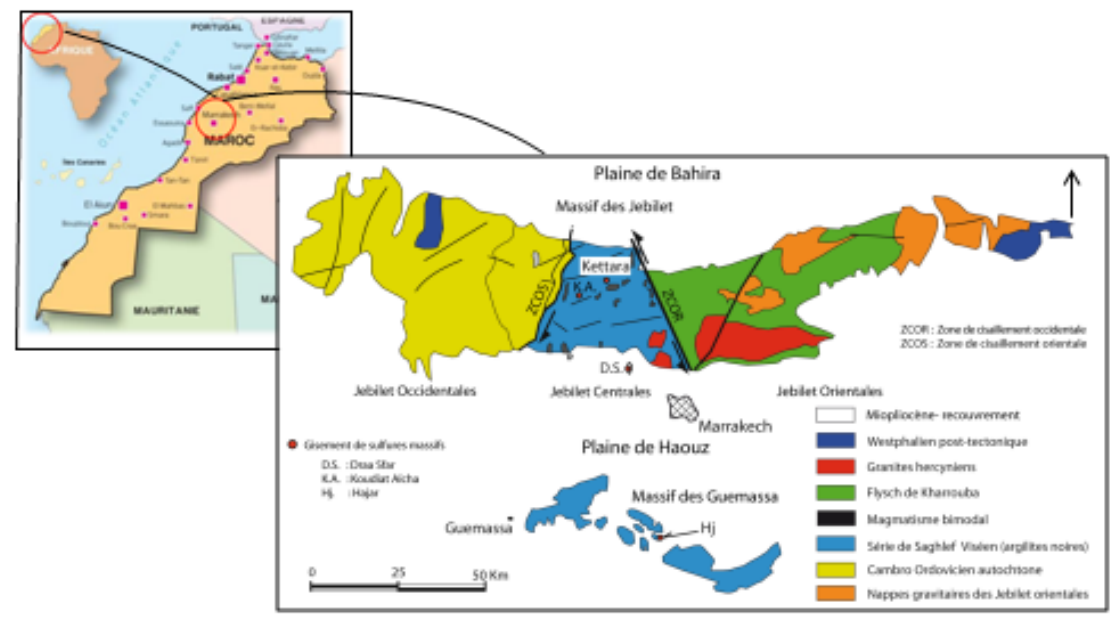

Figure 1 : Situation géologique du site de Kettara (Marcoux et al., 2008, modifiée in Leghoul 2014)

La caractérisation des résidus miniers et des stériles de Kettara a fait l'objet des travaux de Hakkou et al. (2008a, b) ; Nfissi et al. (2011) et Nfissi (2013) et a montré que le $\mathrm{pH}$ des lixiviats produits par ces résidus sont très acides $(\mathrm{pH}<3)$. Les valeurs de $\mathrm{pH}$ sont comparables à celles des eaux de 
ruissellement prélevées in situ (Ouakibi et al., 2013). Les tests de perméabilité sur les échantillons des résidus ont fourni des coefficients de perméabilité compris entre $4,9 \times 10^{-2} \mathrm{~cm} / \mathrm{s}$ et $1,1 \times 10^{-1} \mathrm{~cm} / \mathrm{s}$ ce qui permet de les classer parmi les matériaux parfaitement perméables (Nfissi, 2013). Les mesures du potentiel net de génération d'acide de Kettara ont fourni des valeurs comprises entre 51 et $453 \mathrm{~kg} \mathrm{CaCO}_{3} / \mathrm{t}$ alors que le potentiel net de neutralisation reste faible et varie entre - 453 à - $22.5 \mathrm{~kg} \mathrm{CaCO}_{3} / \mathrm{t}$ (Hakkou et al., 2008a).

Les phases minéralogiques dominantes sont constituées de pyrrhotite et de pyrite accompagnées par de petites quantités de chalcopyrite, de sphalérite, de goethite, d'aluminosilicates, de chlorite-serpentine, de quartz, de talc et de muscovite. Le pourcentage des carbonates des résidus de Kettara, déterminé par calcimétrie de Bernard, est de l'ordre de $2.95 \%$. Ce faible pourcentage n'agira pas sur la neutralisation du milieu et la teneur en matière organique dans ces résidus, ne dépasse pas $1 \%$.

Ces résidus constituent donc un stock important de métaux toxiques et d'acidité qui contaminent les sols, les eaux de surface et souterraines et portent atteinte à la santé humaine. Cette menace est matérialisée par le déclanchement du drainage minier acide (DMA). La réhabilitation de cette mine et l'atténuation de ce phénomène sont fortement recommandées.

\section{2- Matériaux et méthodes analytiques}

\section{2-1- Les boues de sucrerie (MPS)}

Les boues de pâte de sucrerie utilisées dans le cadre de cette étude proviennent de la station d'épuration des eaux usées de la COSUMAR qui est considérée comme étant l'une des plus grandes raffineries d'Afrique. Cette dernière se charge de la transformation du sucre brut en sucre raffiné sous quatre formes: Pains de sucre, lingots, morceaux et granulé (Figure 2).

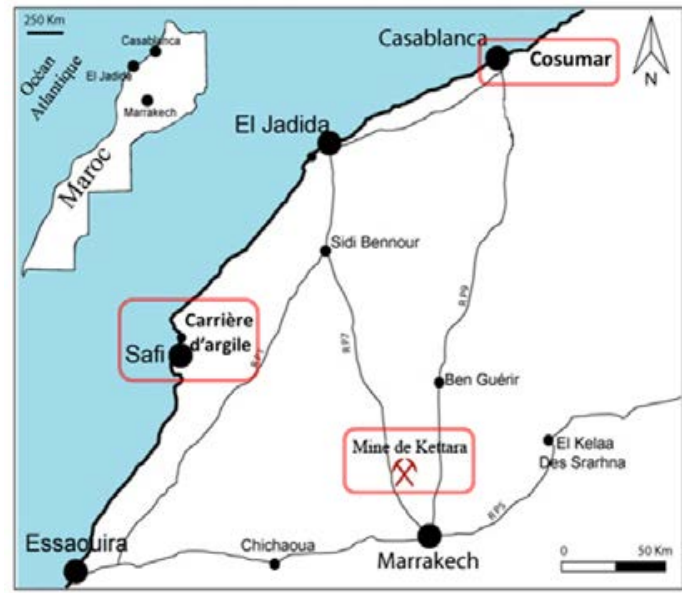

Figure 2 : Localisation des différents sites de collecte de matériaux utilisés (MPS et CLY) (Nfissi et al., 2014) 


\section{2-2 Les argiles (CLY)}

Les argiles utilisées dans cette étude ont été prélevées d'une carrière qui se situe à une dizaine de Km à l'Est de la ville de Safi (Figure 2). Les argiles hauteriviennes de Safi forment un bassin étendu, rectangulaire, de 5 km de longueur sur $3 \mathrm{~km}$ de largeur. Ces argiles, exploitées principalement pour la poterie (Photo 1), reposent sur un calcaire jaune en plaquettes attribué au Valanginien. Elles sont surmontées par une épaisse série de calcaire blanc cristallin qui date de l'Hauterivien supérieur (El Yakoubi, 2006).

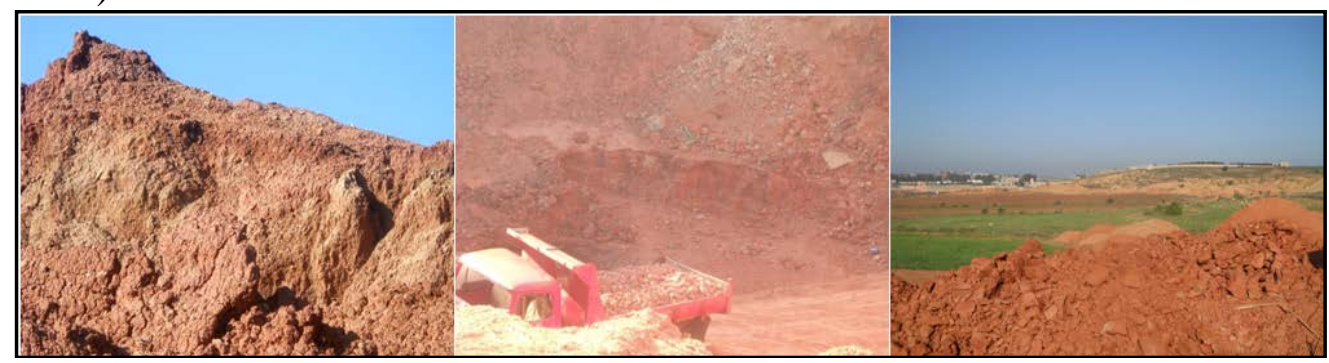

Photo 1: Photos illustrant la carrière des argiles rouges de Safi.

\section{3- Méthodes analytiques}

Le test statique le plus couramment utilisé afin de prédire le potentiel de production d'acide est la méthode standard appelée : Acid-Base Accounting (ABA). Elle a été développée par Smith et al. (1974) et modifiée par différents auteurs (Sobek et al., 1978; Lawrence et Wang, 1997; Bouzahzah, 2013). Le potentiel de neutralisation (PN), exprimé en kg équivalent $\mathrm{CaCO}_{3} / \mathrm{t}$., des échantillons utilisés est déterminé par une digestion à l'acide chlorhydrique (1N) (Bouzahzah, 2013). La minéralogie quantitative par diffractométrie aux rayons $X$ (DRX) fut établie au sein de l'Unité de recherche et de service en technologie minérale (URSTM) au Canada. Le pourcentage des carbonates des matériaux étudiés a été déterminé par le calcimètre de Bernard (Chamely, 1966) et le dosage de la matière organique, a été effectuée selon la méthode de (Walkley et Black, 1934), dans la salle d’analyses du département de Géologie (Faculté des Sciences Ben M’Sik de Casablanca).

\section{4- Résultats et discussion}

\section{4-1- Propriétés physico-chimiques et minéralogiques des tests statiques des MPS}

En adoptant la méthode standard test acide-base (ABA) de Sobek et al., (1978) modifiée, le potentiel de neutralisation de la boue de la pâte de sucrerie est de l'ordre de $878.5 \mathrm{~kg} / \mathrm{t} \mathrm{CaCO}_{3}$; il est plus élevé que celui des déchets des phosphates alcalins qui est de l'ordre de $680 \mathrm{~kg} / \mathrm{t} \mathrm{CaCO}_{3}$ (Hakkou et al., 2009) et est cependant inférieur à celui des poussières de four 
de cimenterie qui est de l'ordre de $910 \mathrm{~kg} / \mathrm{t} \mathrm{CaCO}_{3}$ (Nfissi et al., 2014). Ce fort potentiel de neutralisation reflète leur forte alcalinité qui permettra de neutraliser l'acidité des résidus miniers de Kettara.

La principale phase détectée par diffractométrie aux rayons $\mathrm{X}$ des boues de sucrerie est la calcite (94\%). Le quartz, présent dans ces boues, ne dépasse pas 3.2\% (Figure 3).

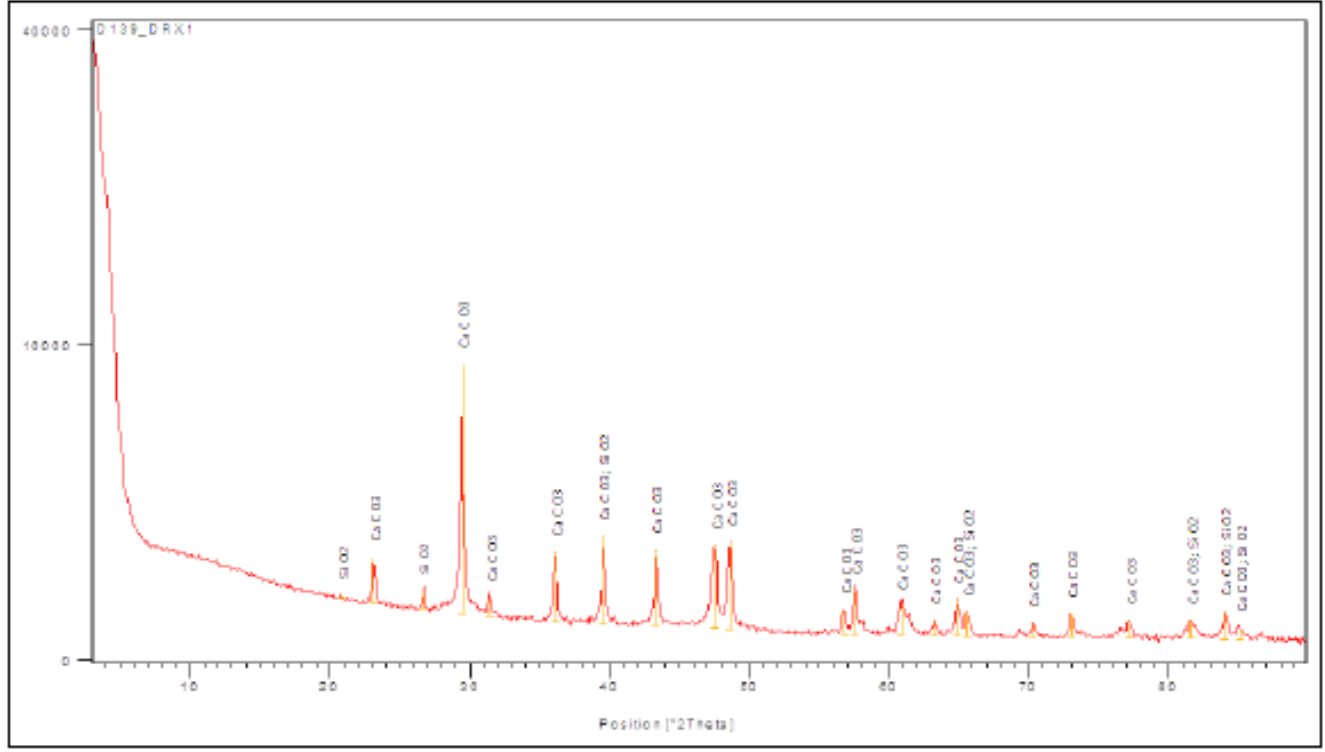

Figure 3: Diffractométrie aux rayons $\mathrm{X}$ des boues de sucrerie

En ce qui concerne l'évaluation du taux de matière organique, les résultats obtenus montrent que sa teneur dans les boues de la pâte de sucrerie est de l'ordre de $8.35 \%$ (Tableau I). Ce taux est relativement faible par rapport à la matière minérale qu'elle contient. Cette proportion est identique à celle obtenue par Sahnoun (2011).

La teneur en carbonate de calcium dans la boue de filtration est en moyenne de $80 \%$ (Tableau I). Ceci concorde parfaitement avec la proportion de la calcite obtenue par DRX.

\begin{tabular}{lll}
\hline Echantillons & M.O. (\%) & $\mathrm{CaCO}_{3}(\%)$ \\
\hline MPS & $\mathbf{8 . 3 5}$ & $\mathbf{8 0}$ \\
\hline
\end{tabular}

Tableau I : Dosage de la matière organique et des carbonates dans les boues de la pâte de sucrerie.

\section{4-2- Propriétés physico-chimiques et minéralogiques des tests statiques des CLY}

Les résultats du test acide-base 'ABA' (Acid-Base Accounting) modifiée par Sobek et al., (1978), ont montré que le potentiel de neutralisation des argiles de Safi est de l'ordre de $299 \mathrm{~kg} / \mathrm{t} \mathrm{CaCO}_{3}$. Ce 
pouvoir est relativement faible par rapport à celui des poussières de four de cimenterie $\left(910 \mathrm{~kg} / \mathrm{t} \mathrm{CaCO}{ }_{3}\right)$ et avoisine celui des cendres volantes $(250 \mathrm{~kg} / \mathrm{t}$ $\mathrm{CaCO}_{3}$ ) (Nfissi et al., 2014). Malgré l'alcalinité modérée de ces argiles, par rapport à celles des CKD et des MPS, ils peuvent cependant contribuer à la neutralisation de l'acidité des résidus miniers de Kettara.

Le spectre de DRX des argiles révèle la présence des phases minérales argileuses constituées de kaolinite et de muscovite, auxquelles sont associées des phases minérales distinctes à savoir le quartz et la calcite (Figure 4).

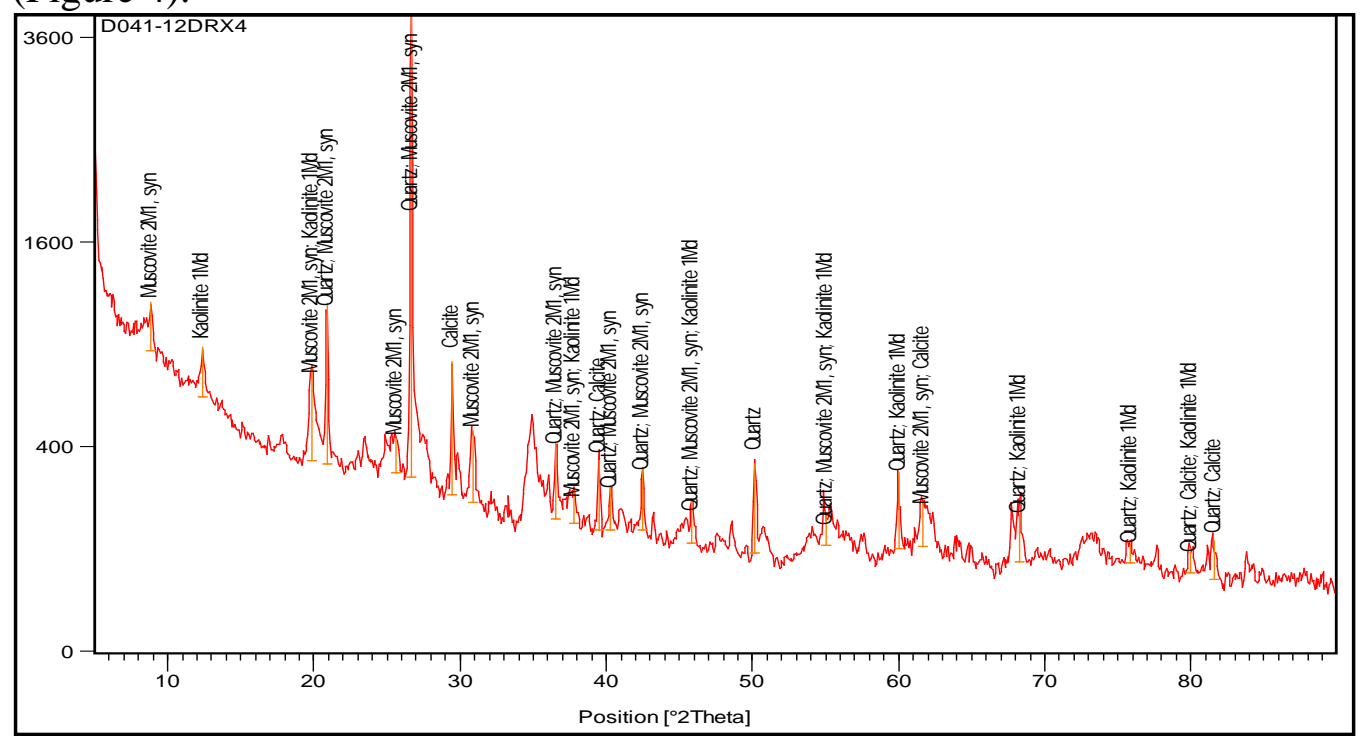

Figure 4 : Diffractométrie aux rayons X des argiles rouges de Safi

Les échantillons des argiles analysées montrent un pourcentage de la matière organique de l'ordre de $0.53 \%$ (Tableau II). Cette teneur en matière organique, est négligeable par rapport à celle des boues de la pâte sucrière. La teneur en carbonate de calcium dans les argiles de Safi est en moyenne de $10.48 \%$ (Tableau II).

\begin{tabular}{lll}
\hline Echantillons & M.O. (\%) & $\mathrm{CaCO}_{3}(\%)$ \\
\hline CLY & 0.53 & 10.48 \\
\hline
\end{tabular}

Tableau II : Dosage de la matière organique et des carbonates dans les argiles de Safi.

\section{5- Conclusion}

Les MPS sont composées de calcite et de quartz; les argiles, de kaolinite, de quartz, de calcite et de muscovite.

L'ensemble des analyses effectuées ont montré que les boues de pâte de sucrerie sont dotées d'un potentiel de neutralisation élevé qui est de l'ordre de $878.5 \mathrm{~kg} / \mathrm{t} \mathrm{CaCO}_{3}$. Celui des argiles et qui est de l'ordre de 299 
$\mathrm{kg} / \mathrm{t} \mathrm{CaCO}_{3}$ ), reste modéré par rapport à celui des boues de sucrerie, mais participeront pour autant à la neutralisation de l'acidité des résidus miniers.

Selon Parker \& Rea (1989), les argiles sont utilisées dans la construction de barrières imperméables. Elles peuvent être utilisées seules ou mélangées avec d'autres matériaux.

Les boues de sucrerie et les argiles sont donc considérées comme étant des matériaux appropriés pour la stabilisation des rejets miniers acides ; les argiles serviront de barrière pour l'infiltration.

Grâce aux résultats obtenus, des tests cinétiques seront conduits au laboratoire sur des essais en mini-cellules pour suivre l'évolution de la qualité du lixiviat acide produit par les résidus miniers amendés par un mélange de MPS et de CLY. L’objectif étant de définir les proportions optimales qui permettront d'atténuer voir même de stopper le DMA produit dans ce site.

\section{Remerciements}

Les auteurs tiennent à remercier les organismes subventionnaires de cette étude; en particulier COSUMAR, le Centre National de la Recherche Scientifique et Technique (CNRST, Rabat) et le laboratoire de Géodynamique des chaines anciennes (FSBM, Casablanca).

\section{References:}

Aubertin M., Bussière B., Bernier L., Chapuis R., Julien M., Belem T., Simon R., Mbonimpa M., Benzaazoua M. \& Li L. (2002) -‘La gestion des rejets miniers dans un contexte de développement durable et de protection de l'environnement'. Congrès annuel de la Société canadienne de génie civil. Montréal, Québec, Canada, 5-8 juin 2002. Paper No.GE-045 /Article No. GE-045, pp.33

Benzaazoua M. \& Kongolo M. (2003) - 'Physico-chemical properties of tailing slurries during environmental desulphurization by froth flotation' International Journal of Mineral Processing, 69: 221-234.

Bordonaro M. (1984) - La ceinture ibéro-marocaine. Géologie structurale du district de Kettara, thèse de $3^{\text {ème }}$ cycle, Strasbourg.

Bossé B. (2013) - 'Évaluation du comportement hydrogéologique d'un recouvrement alternatif constitué de rejets calcaires phosphatés en climat semi-aride à aride'. Ph.D. Diss., UQAT, Rouyn-Noranda, Canada. 301p.

Bouzahzah H. (2013) - 'Modification et amélioration des tests statiques et cinétiques pour une prédiction fiable et sécuritaire du drainage minier acide’. Thèse de Doctorat, Université du Québec en Abitibi-Temiscamingue (UQAT). Aôut 2013, pp. 11-130 et 288.

Chamley H. (1966) - Guide des techniques du laboratoire de Géologie Marine de Luminy , 198p. 
Dagenais A.M., Aubertin M., Bussière B., Cyr G. \& Fontaine R. (2003) Auscultation et suivi du recouvrement multicouche construit au site minier Loraine, Latulipe, Québec. Symposium sur l'Environnement et les mines, Rouyn-Noranda, Québec, Canada. 3-5 novembre.

El Yakoubi N. (2006) - Potentialités d'utilisation des argiles marocaines dans l'industrie céramique : cas des gisements de Jbel Kharrou et de Benhmed (Meseta marocaine occidentale). Thèse de doctorat. Universite mohammed v-agdal. Faculté des sciences Rabat 212 p.

Felenc J., Fournier M., Hmeuras M. (1986) - Contrôles géologiques des amas à pyrrhotine des Jebilet et Guemassa. Définition des guides de recherche. Rap. BRGM 86 MAR 131, 54 p., 29 fig., 2 ann.

González V., García I., Del Moral F., Simón M. (2012) - 'Effectiveness of amendments on the spread and phytotoxicity of contaminants in metalarsenic polluted soil', Journal of Hazardous Materials, pp 74-78.

Hakkou R., Benzaazoua M. \& Bussière B. (2009) - 'Laboratory evaluation of the use of alkaline phosphate wastes for the control of acidic mine drainage'. Mine Water Environ 28:206-218, pp 3-11.

Hakkou R., Benzaazoua M. \& Bussière B. (2008b) - 'Acid Mine Drainage at the Abandoned Kettara Mine (Morocco): 2. Mine Waste Geochemical Behavior', Mine Water Environ 27:160-170. pp. 3-10.

Hakkou R., Benzaazoua M. \& Bussière B. (2008a) - 'Acid Mine Drainage at the Abandoned Kettara Mine (Morocco): 1.Environmental Characterization’, Mine Water Environ 27:145-159. pp. 5-8.

Lawrence R.W. \& Wang Y. (1997) - 'Determination of neutralization potential in the prediction of acid rock drainage', In: Proceedings of 4th International Conference on Acid Rock Drainage, Vancouver, B.C., Canada. pp. 449-464.

Lghoul M. (2014) -‘Apport de la géophysique, de l’hydrogéochimie et de la modélisation du transfert du drainage minier acide au projet de réhabilitation de la mine abandonnée de Kettara (région de Marrakech, Maroc)’. Université Pierre et Marie Curie et Université Cadi Ayyad. ED398 Géosciences et Ressources Naturelles.

Marcoux E., Belkabir A., Gibson H.L., Lentz D. \& Ruffet G. (2008) - 'Draa Sfar, Morocco: A Visean (331 Ma) pyrrhotite-rich, polymetallic volcanogenic massive sulphide deposit in a Hercynian sediment-dominant terrane’. Ore Geology Reviews, 33, 307-328.

Mermillod-Blondin R., Kongolo M., De Donato P., Benzaazoua M., Barrès O., Bussière B. \& Aubertin M. (2005) - 'Pyrite flotation with xanthate under alkaline conditions- application to environmental desulfurization’. Centenary of Flotation Symposium, Brisbane, QLD, 6-9 June 2005.

Nfissi S., Alikouss S., Zerhouni Y., Hakkou R., Benzaazoua M., Samir M. \& Baroudi Z. (2014) -'Evaluation of the capacity of cement kiln dust and fly 
ash for the neutralization of acidic mine tailings'. (MSNo. JGEE 2014/04/02/007), International Journal of Geology, Earth and Environmental Sciences. 2277-2081 (Online), 2014 Vol. 4(2) May-August, pp. 51-61.

Nfissi S. (2013) - 'Evaluation de la capacité de matériaux industriels alcalins à neutraliser les rejets miniers acides de la mine de Kettara (Jebilets Centrales, Maroc)'. Thèse de Doctorat, Université Hassan II Mohammedia Casablanca. FSBM, Décembre 2013.

Nfissi S., Zerhouni Y., Benzaazoua M., Alikouss S., Chtaini A., Hakkou R. \& Samir M. (2011) - 'Caractérisation des résidus miniers des mines abandonnées de Kettara et de Roc Blanc (Jebilet centrales, Maroc)'. Ann. Soc. Géol. du Nord., T. 18 (2 ${ }^{\text {ème }}$ série), pp. 43-53.

Ouakibi O., Loqman S., Hakkou R. \& Benzaazoua M. (2013) - 'The Potential Use of Phosphatic Limestone Wastes in the Passive Treatment of AMD: A Laboratory Study', Mine Water and the Environment (Journal of the International Mine Water Association (IMWA)), pp.5-10.

Parker \& Rea. (1989) - In Réseau Eco-consommation, Jean-François Rixen, Centre Urbain, Evere, 29 octobre 2008. La chaux et l'argile : du sol au plafond.

Plante M. (2004) - 'Comparaison des essais statiques et évaluation de l'effet de l'altération pour des rejets de concentrateur à faible potentielde génération d'acide’. Mémoire de maîtrise, École Polytechnique de Montréal, pp.241.

Sahnoun E.M. (2011) - 'Caractérisation physique-chimique et étude de valorisation des boues issues de l'unité de filtration de l'industrie sucrière'. Mémoire de Master Univ. Hassan II Mohammedia-Casablanca, Fac. Sci., Ben M’sik.48p.

Smith R.M., Grube W.E., Arkle T. \& Sobek A. (1974) - 'Mine Spoil Potentials for Soil and Water Quality'. U.S. Environmental Protection Agency, Cincinnati, Ohio, EPA-670/2-74-070, 303p.

Sobek A. A., Schuller W. A., Freeman J. R. \& Smith R. M. (1978) - 'Field and laboratory methods applicable to overburdens and minesoils', EPA600/2-78-054, U.S. Gov. Print. Office, Washington, DC.

Walkley A. \& Black I.A. (1934) - 'An examination of the Degtjareff method for determining organic carbon in soils: Effect of variations in digestion conditions and of inorganic soil constituents', Soil Sci. 63:251-263. 